\title{
LAS BASES SOCIALES DE LA ENFERMEDAD MENTAL
}

Benjamín González Rodríguez

El tema de la enfermedad mental resulta complejo, tanto por lo que se refiere a su origen como a sus manifestaciones y consecuencias. Analizamos en este trabajo las bases sociales de la etiología de la enfermedad mental. El interrogante básico consiste en saber si los factores sociales pueden «causar» o no la enfermedad mental. La pregunta no es ni reciente ni sencilla de responder, ya que, como señala Rosenthal (1970:186): «Son tantas las causas ambientales, bioquímicas o psicológicas que a lo largo de los años se han postulado, que resulta casi más fácil enumerar los factores que no han sido propuestos que los que sí lo han sido." La discusión que sigue se organiza en torno a los cuatro puntos siguientes:

- Evolución histórica de los conceptos etiológicos.

- Revisión de los estudios publicados sobre las bases sociales de la enfermedad mental.

- Cambios necesarios y urgentes en esta línea de investigación.

- Dirección futura de la investigación sobre las bases sociales de la enfermedad mental. 
Evolución bistórica de los conceptos etiológicos

Los conceptos etiológicos han fluctuado siguiendo en paralelo los avances médicos. Existen tres estadios básicos en estos avances y fluctuaciones históricas. Hipócrates tuvo en consideración el concepto de ambiente relacionándolo con el de enfermedad. Esta relación obedecía a la necesidad de claridad en la conceptualización de las variables y de los modelos de causalidad. Tal como señala Susser (1973:14), los avances producidos en medicina y en sanidad pública se debieron a esta claridad de conceptualización. Baste citar, en este sentido, el ejemplo de la malaria. Los primeros ambientalistas estaban en lo cierto al distinguir entre ambiente (agua, aire, etc.) y huésped (individuo, constitución, etc.). Fue, sin embargo, en el siglo xIX cuando se lograron avances realmente importantes. Uno de los precursores de estos avances fue John Graunt, en Londres. Desde el punto de vista cuantitativo, hay que mencionar a E. Chadwick (1842), con su Report on an Inquiry into the Sanitary Conditions of the Labouring Population of Great Britain. Dentro de los aspectos etiológicos, Pasteur abre el segundo estadio, al introducir los dos conceptos fundamentales de inmunidad y huésped. El interés de Pasteur se centraba especialmente en las manifestaciones de los trastornos más bien que en la capacidad o posibilidades del huésped para afrontar y configurar tales trastornos. De otro lado, y dentro de este segundo estadio, cabe mencionar a Darwin y a Mendell, quienes insistían en que tanto los atributos heredados como los adquiridos pueden determinar el trastorno del huésped. Tanto Darwin como Mendell enfatizaron la importancia de los factores del huésped para contraer la enfermedad. Finalmente, el tercer estadio consistió en tratar de explicar los factores ambientales que inciden en las enfermedades (infecciosas). En esta etapa, los investigadores tuvieron que enfrentarse con la compleja tarea de sintetizar la tríada formada por el agente transmisor, el huésped u organismo receptor de la enfermedad, y el ambiente. Al intentar esta síntesis, el modelo ecológico descubre pautas complejas de interacción entre los componentes de la tríada. Es decir, ya no se puede concebir la enfermedad unilateralmente en cuanto que uno de los elementos de la tríada sea el responsable del trastorno, sino que en el nacimiento $u$ origen de la enfermedad, los tres componentes interactúan de modo complejo. No obstante, incluso este modelo ecológico carecía de la precisión suficiente como para poder descubrir relaciones de tipo causal. Dado que, como hemos indicado, los elementos de la tríada interactúan, el epidemiólogo necesita explicaciones de causalidad múltiple de la enfermedad (Susser, 1973).

Resumiendo, tendríamos tres estadios básicos en el desarrollo de los conceptos etiológicos. Se considera, en primer lugar, que existen múltiples factores responsables de la enfermedad. Posteriormente, aparece el concepto de etiología especifica, es decir, el estudio de los agentes específicos pro- 
ductores de la enfermedad, como sucedió en el caso de las enfermedades infecciosas. Finalmente, surgió la idea de la etiología múltiple, según la cual se tiene en cuenta una especie de constelaciones de condiciones que serían las responsables de la aparición de la enfermedad. Esta etiología múltiple necesita de un enfoque más amplio que el de las dos fases precedentes para poder explicar enfermedades tales como el cáncer, los trastornos coronarios, la diabetes, los accidentes y la enfermedad mental, entre otras (Mechanic, 1968).

La razón por la que hemos delineado estos estadios históricos está en que muchos investigadores, al tratar el tema de la etiología de la enfermedad mental, han utilizado y aplicado modelos válidos para las enfermedades infecciosas a las no-infecciosas, tales como la enfermedad mental. En este último caso, la búsqueda, por ejemplo, de agentes específicos puede resultar equivocada y/o llevarnos a callejones sin salida, porque, tal como han demostrado muchas investigaciones en este área, existen, de hecho, muchas posibles causas de la enfermedad mental. Si bien es cierto que podemos encontrar o descubrir una condición necesaria para que surja la enfermedad mental, sin embargo, la pregunta decisiva podría formularse de la siguiente manera: ¿resulta suficiente esta condición necesaria para explicar la aparición de la enfermedad mental? Tal como veremos seguidamente al revisar los estudios realizados, no parece verosímil suponer que la condición necesaria, caso de existir, sea suficiente para explicar la enfermedad mental.

\section{Los estudios realizados}

Destaquemos, en primer término, que los estudios producidos en las cuatro últimas décadas son muy numerosos, por lo que nos limitaremos a considerar algunas investigaciones concretas sobre los factores etiológicos (sociales) de la enfermedad mental. Ha habido, históricamente, un conflicto entre lo que podríamos denominar postura biológica o biogénica y postura sociogénica. En todo caso, ambas se refieren fundamentalmente a problemas etiológicos (Price, 1974). Hay que destacar también el hecho de que las medidas de acción concreta, sobre todo desde el punto de vista de la prevención, están mediatizadas por la postura que se adopte frente al conflicto biogenético-social, ya que las medidas preventivas serán distintas según se considere la enfermedad como algo causado desde el interior del individuo, o, por el contrario, desde el medio ambiente social.

El concepto de etiología específica se refiere a la condición causal que es necesaria pero no suficiente para que aparezca la enfermedad (Susser, 1973; Kohn, 1970). Según esto, el agente específico ha de estar presente en la enfermedad, pero su mera presencia no necesariamente determina o produce la aparición de la misma. Tal como señalamos más arriba, pueden 
surgir serios inconvenientes y malentendidos cuando se aplica el concepto de etiología específica a los trastornos mentales; por ejemplo, a la esquizofrenia ${ }^{1}$ (Meehl, 1962). Más específicamente, Price $(1974: 290)$ ha resumido estos malentendidos del modo siguiente: la presencia del factor etiológico no produce necesariamente el trastorno clínicamente observable; de otra parte, la mayor fuente de variación de síntomas no se debe necesariamente a la etiología específica. Además, la etiología específica no implica una sim. ple cadena causa-efecto. En consecuencia, pueden existir numerosos lazos me. diadores entre los factores etiológicos y los síntomas manifiestos; por esta razón, incluso en aquellos trastornos con una etiología específica de origen genético, los factores sociales pueden determinar, en gran parte, el hecho de que aparezca o no el trastorno en cuestión. En este sentido, Price (1974) destaca el hecho de que, si bien sabemos que los factores genéticos contribuyen considerablemente al desarrollo de la enfermedad de la esquizofrenia, sin embargo, los estudios con gemelos demuestran que los factores no-genéticos juegan también un papel importante.

Rosenthal (1970) ha sometido a crítica los numerosos estudios que han tratado de explicar la etiología de la esquizofrenia y de otros trastornos mentales, basándose en variables socio-culturales y socio-económicas. Tras revisar una serie de estos estudios epidemiológicos, presenta un resumen de las diferentes explicaciones ofrecidas por los distintos autores. Según una de estas explicaciones, la pobreza, la educación, el status socio-económico y las relaciones familiares de las clases sociales bajas pueden "causar" o suscitar la esquizofrenia. Otra explicación que se ha dado es que los esquizofrénicos «van a parar» a los suburbios de las grandes ciudades, donde encuentran el anonimato y la tolerancia por parte de los demás. Otra interpretación alternativa sugiere que las familias portadoras de los genes de la esquizofrenia son menos capaces de afrontar los problemas sociales y económicos; tienen, por otra parte, una movilidad ascendente menor, $y$, en consecuencia, tienden a concentrarse en la base de la escala social. Finalmente, otros autores propugnan que en las clases sociales más bajas el cuidado prenatal es más pobre y escaso, lo que aumentaría las tasas de esquizofrenia.

La crítica de Rosenthal a estas explicaciones alternativas es que ninguna de ellas ha logrado demostrar cuál de las hipótesis anteriores es verdadera con respecto a las demás. Nuestro punto de vista es que la eterna controversia de factores biogénicos versus factores sociales está incorrectamente planteada, sobre todo, si tenemos en cuenta el aspecto decisivo de la causación múltiple de la enfermedad mental. Así, por ejemplo, Fried (1976) ha enfocado el problema en términos de una serie de variables intervinientes que median la relación entre clase social y esquizofrenia:

' En el resto de este trabajo nos referiremos fundamentalmente al caso de la esquizofrenia, ya que es éste el trastorno que más se ha estudiado. 
Relación entre clase social y esquizotrenia

\begin{tabular}{lll}
\hline $\begin{array}{c}\text { Variable } \\
\text { independiente }\end{array}$ & \multicolumn{1}{c}{$\begin{array}{c}\text { Variables } \\
\text { intervinientes }\end{array}$} & $\begin{array}{c}\text { Variable } \\
\text { dependiente }\end{array}$ \\
\hline Clase social. & Raza. & Esquizotrenia. \\
& Emigración y disrupción & \\
& social. \\
& Minorías. \\
& Estado civil. \\
& Desempleo. \\
& Movilidad social. \\
\hline
\end{tabular}

Fried considera que resulta difícil valorar y precisar la dirección de la causalidad y concluye que estas variables intervinientes ejercen un impacto acumulativo sobre los determinantes de los trastornos psicológicos. Al hablar del estudio del Midtown Manbattan dice:

«Ninguna experiencia única implicaba el ser considerada como causa principal o como acontecimiento único precipitante. Puede ser, por tanto, que la expectativa habitual de que un solo factor importante vaya a explicar una gran parte de la varianza resulte inadecuada para investigar los trastornos psiquiátricos; puede también darse el caso de que una serie de factores distintos tengan efectos determinantes menores $e$ independientes, y que sólo su efecto acumulativo se convierta en un determinante potente y muy general de la enfermedad mental» (Price, 1976:187).

Lo que parece probable es, pues, que si bien una serie de factores sociales aislados pueden no ejercer un impacto significativo sobre la enfermedad mental, sin embargo, su impacto conjunto sí que puede influir significativamente, a través de mecanismos cuyo efecto se ha ido acumulando. Aquí entra en juego el fenómeno de la interacción, en el sentido de que, además del efecto aislado de cada variable independiente sobre la dependiente hay que tener en cuenta el impacto conjunto de dos o más variables independientes sobre la variable dependiente.

Siguiendo ahora con las explicaciones dadas a la enfermedad mental, Dunham (1970) ha aislado siete hipótesis que compiten entre sí y que tratan de aclarar la relación existente entre los distintos factores del sistema social y la esquizofrenia. La primera hipótesis es la de la anomía y/o desorganización social. Esta hipótesis es la más antigua y predominó entre las dos guerras mundiales. Trata de explicar la distribución diferencial de diversos fenómenos de conducta en términos de carencia de normas, falta de control 
y decadencia de la cohesión social. La base de esta hipótesis está en las teorías de Durkheim para quien el individualismo excesivo y el relajamiento del control social serían la causa de los trastornos de conducta. La crítica a esta hipótesis consiste en que los conceptos que utiliza resultan vagos y no nos dicen, en realidad, nada sobre la etiología y la distribución de la esquizofrenia en los sistemas sociales. Otra explicación proviene de la hipótesis del drift, según la cual, la variación de las tasas de esquizofrenia en distintas regiones contiguas se debe a una acumulación inconsciente de las personas enfermas en áreas en las que las posiciones o status no están tan delimitadas como en las áreas de las que provienen estas personas. El primero que propuso esta hipótesis fue Myerson (1941) al tratar de descubrir los distintos tipos de distribución de la esquizofrenia en Chicago. Lo que Myerson pretendía era explicar los resultados del trabajo de Faris y Dunham (1939). Los críticos principales de esta hipótesis han sido, entre otros, Faris y Dunham (1939), y Hollingshead y Redlich (1958). Sus críticas pueden resumirse en los puntos siguientes: la imagen que da esta hipótesis del esquizofrénico es la de una persona incoherente, impulsiva, confusa y desconocedora de lo que hace y de por qué lo hace; asimismo, la hipótesis no tiene en cuenta que los primeros pasos hacia la enfermedad los da la familia misma del paciente, y, por último, niega al paciente cualquier capacidad o posibilidad de elección voluntaria en su vida. La tercera hipótesis es la de la movilidad. Está ligada a las dos anteriores y puede formularse así: ¿tienen los enfermos una mayor o menor movilidad que las personas normales? Los resultados empíricos son contradictorios. Otro planteamiento sería: ¿tienen los enfermos una mayor movilidad geográfica o es que la movilidad acelera o facilita la esquizofrenia? Tampoco aquí los datos empíricos existentes permiten generalizar. En consecuencia, la movilidad per se no parece constituir una explicación importante de la variación de tasas de la esquizofrenia.

Otra hipótesis es la de los distintos niveles de tolerancia por parte de la comunidad. Se ha utilizado para explicar las distintas tasas de enfermos mentales en zonas rurales y urbanas, así como los distintos niveles de aceptación de la enfermedad mental por parte de las familias de clase alta y baja. La hipótesis sostiene que una actitud favorable y comprensiva hacia el enfermo mental hace que éste no sea hospitalizado, y, en consecuencia, al no pasar por el hospital, no es contado o no figura en las estadísticas. Por el contrario, la no-aceptación llevaría a una hospitalización más rápida y, por tanto, a que el enfermo figure en las estadísticas. Otra de las hipótesis es la del aislamiento voluntario, es decir, los esquizofrénicos se aíslan ellos mismos eligiendo ciertas zonas para residir, zonas que les permitan no tener que mantener relaciones inter-personales. De hecho, algunos estudios han correlacionado el hecho de vivir solo con la esquizofrenia. La sexta hipótesis es la de la cobesión social. En realidad, es la contraria de la anterior. La idea, en este caso, es que allí donde existe un alto grado de cohesión social, 
moral y buenas relaciones inter-personales, cabe esperar una alta incidencia de psicosis maníaco-depresivas. Esta hipótesis se basa en el estudio de Eaton y Weil sobre las comunidades huteritas. Finalmente, la hipótesis del tamaño de la ciudad considera las variaciones de incidencia de la esquizofrenia como un producto de las diferencias demográficas entre poblaciones, y no como el resultado de los factores sociales y culturales que estarían presentes en unas áreas y ausentes en otras. Según esto, la hipótesis sería que a medida que aumenta el tamaño de la ciudad, la tasa de esquizofrenia de las distintas áreas se hace más diferenciada. En opinión de Dunham, ninguna de las siete hipótesis precedentes logra explicar las tasas distintas de esquizofrenia en las grandes ciudades.

No obstante, la opinión de Mischler y Scotch (1970) resulta algo más optimista que la de Dunham. Tras revisar nueve estudios sobre tasas de incidencia de la esquizofrenia, encontraron algunos resultados consistentes, como, por ejemplo, que la incidencia va asociada a los estratos sociales inferiores, $y$, por el contrario, la tasa menor de incidencia va asociada con los grupos directivos de la sociedad. Sin embargo, a pesar de estos resultados consistentes, caben explicaciones alternativas de estas relaciones. Existe, por una parte, la posibilidad de que las correlaciones encontradas sean espúreas, y, por otra, puede haber en estas relaciones diversas fuentes de error debidas a la medición de las variables, a la identificación de los casos, y a los procedimientos de diagnóstico.

Por su parte, Melvin Kohn (1970) lleva a cabo una revisión más valiosa del problema. Destaca este autor la importancia de la susceptibilidad genética, a la vez que pone el énfasis en el papel de la familia como transmisora de las distintas concepciones de la realidad social. Según esta perspectiva, las clases sociales más bajas transmiten a su descendencia puntos de vista excesivamente estrechos y rígidos como para poder manejar con eficacia y con éxito las situaciones de stress. La interpretación de Kohn nos parece más útil porque tiene en cuenta un tipo de causalidad múltiple que abarca la genética, el stress y las distintas condiciones de vida de las diferentes clases sociales, para interpretar de modo coherente la esquizofrenia (Kohn, 1972). El esquema siguiente sintetiza las ideas de este autor:

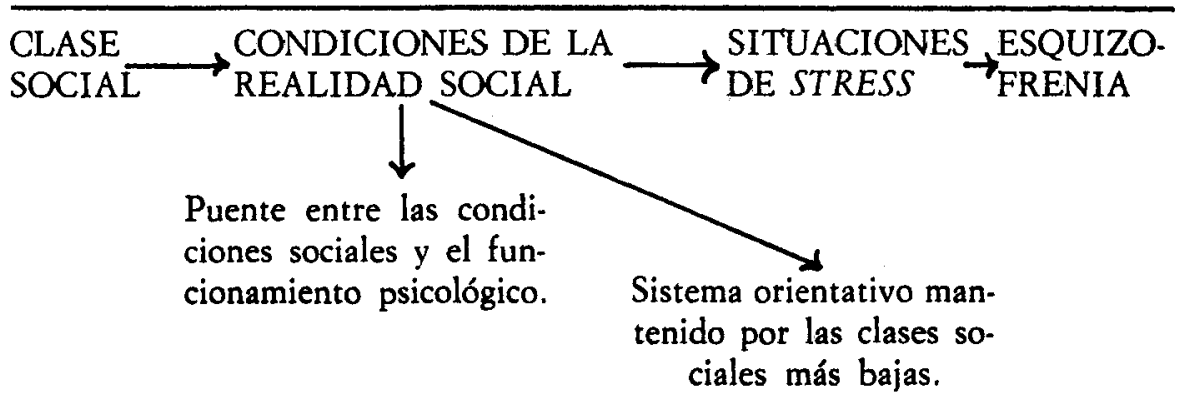


Entre la clase social y la esquizofrenia existen dos tipos de lazos o eslabones; de una parte, las condiciones de la realidad social de los individuos, y de otra, las situaciones de stress producidas por dichas condiciones. Estas últimas cumplen una doble función en cuanto puente entre las situaciones de vida de las distintas clases sociales y su funcionamiento psicológico, y en cuanto que configuran un sistema orientativo más rígido en el caso de las clases sociales más bajas. Estas condiciones de vida distintas para las distintas clases crean situaciones de stress cuyo impacto es también distinto en cada una de las clases. Cabe suponer, asimismo, que las clases sociales más bajas presentan una mayor susceptibilidad al stress. En síntesis, la estratificación social crea unas condiciones de vida que tienen un impacto diferencial sobre el stress y, en consecuencia, sobre el funcionamiento psicológico del individuo.

Lo que se desprende de las múltiples hipótesis alternativas anteriormente expuestas es que no existe un acuerdo entre ellas a la hora de explicar la incidencia y prevalencia de la enfermedad mental. Por ello, algunos autores han tratado de averiguar los fallos subyacentes a este tipo de estudios, proponiendo una serie de críticas a los mismos. Mischler y Scotch (1970) resumen adecuadamente estas críticas. Señalan, en primer lugar, que lo más típico de este área ha sido una gran cantidad de investigación inadecuada junto con numerosas críticas adecuadas. Otra de las características de estos estudios ha consistido en que sus diseños han sido bastante simples, y se han limitado a una mera epidemiología descriptiva. Dentro de las críticas metodológicas, cabe destacar que la estadística utilizada resulta excesivamente elemental; no se han utilizado técnicas multivariadas ni se han realizado estudios de seguimiento de las hipótesis iniciales de investigación. Por otra parte, los índices de morbilidad empleados resultan problemáticos, ya que las correlaciones ecológicas y las individuales arrojan resultados distintos. Asimismo, tanto las variables sociales como las formas de análisis suelen ser bastante simplistas. Se da, de otro lado, una tendencia a utilizar únicamente variables macro-sociales; no se utilizan otras variables a nivel micro, como procesos familiares, condiciones de vida, etc. Tampoco se ha tenido en cuenta la posibilidad de manejar simultáneamente variables macro y micro.

Desde el punto de vista de la enfermedad como tal, se da, en primer lugar, un desconocimiento grave de la enfermedad misma. En segundo lugar, no se tienen en cuenta las distintas sub-clases de esquizofrenia, de forma que pudieran ponerse en relación las variables sociales con cada sub-tipo de esquizofrenia. Existe, finalmente, un grave problema relacionado con la fiabilidad de los procedimientos gnosológicos y de diagnóstico. Otra característica de estos estudios consiste en la ausencia de trabajo en equipo, que facilitaría el enfoque multi-causal del que hemos hablado. Ha existido, de hecho, un aislamiento entre investigadores, teóricos y clínicos. A la vista de estos fallos, la consecuencia inmediata sería un replanteamiento de este tipo 
de estudios para introducir en ellos una serie de modificaciones lógicas, tal como proponemos a continuación.

\section{Cambios necesarios y urgentes}

Nuestro punto de vista es que ha llegado el momento de reducir el enorme caudal de estudios ya existente (estudios repetitivos, en su mayor parte) y de ponerse a pensar. La tarea inmediata consistiría en una re-evaluación y re-planteamiento de los principios y presupuestos básicos de la epidemiología psiquiátrica. Durante las cuatro últimas décadas se ha logrado muy poco en cuanto a etiología y prevención a base de repetir los mismos análisis, sin realizar un examen cuidadoso y detallado de los fines, técnicas y metodología empleadas hasta ahora. Parece como si los investigadores mantuvieran, implícitamente al menos, la hipótesis de que la mera réplica de trabajos hechos en otro lugar y por otros autores, y su acumulación, pudieran producir un salto cualitativo, es decir, el descubrimiento de la etiología específica de la enfermedad mental.

Habría, pues, que introducir una serie de cambios de dirección, necesarios y urgentes a la vez. En primer lugar, habría que prestar mucha más atención a definir con exactitud el papel de la epidemiología en la enfermedad mental. En este contexto cabría preguntarse, por ejemplo, si no sería posible afirmar que el aumento del número de pacientes no refleja más que el aumento del número de psiquiatras. La razón reside en que son precisamente los psiquiatras quienes normalmente definen qué es un caso psiquiátrico y, en definitiva, son ellos también quienes poseen la llave para decidir si la enfermedad mental existe o no. Parece, pues, lógico pensar que cuanto mayor sea el número de «definidores», mayor será también el número de «definidos». En consecuencia, una meta fundamental de la epidemiología psiquiátrica consiste en hallar, si es que ello es posible, una definición exacta de qué es lo que constituye un caso psiquiátrico, ya que éste es, precisamente, su objeto de estudio. Por otra parte, la falta de criterios diagnósticos objetivos y la dificultad seria para determinar el momento exacto en que surge la enfermedad mental, constituyen dos problemas básicos que impiden la comprensión precisa de la verdadera incidencia de la enfermedad mental, lo que, a su vez, hace que resulte prácticamente imposible cualquier descubrimiento adecuado en relación con el problema de la etiología. Esto queda claro si tenemos en cuenta los datos de Dohrenwend y Dohrenwend (1969 y 1970) sobre el alcance o amplitud de la enfermedad mental, que oscila entre menos del 1 por 100 en la población y más del 64 por 100 , según los distintos estudios.

Otro cambio necesario se refiere a la metodología utilizada por los investigadores en el diseño de sus estudios. En general, ha sido muy pobre, en 
cuanto que no se han utilizado métodos de investigación refinados. Así, por ejemplo, los índices de clase social han sido frecuentemente correlacionados con los índices de enfermedad mental, sin que se haya prestado atención a la fiabilidad de tales índices. Muchos investigadores han identificado «clase social» con ingresos económicos, la mayoría con ocupación, y muy pocos se han preocupado por la clase social en cuanto índice de prestigio (o prestancia) social. Se sabe, asimismo, que si bien educación y ocupación están altamente correlacionados, sin embargo, reflejan una estabilidad temporal distinta, en el sentido de que el status social es más estable que la educación, que históricamente ha cambiado más. Por otra parte, cuando se utilizan conjuntamente la educación, la ocupación, los ingresos económicos y el lugar de residencia, no hay forma de aislar los efectos específicos de cada uno de estos índices sobre la enfermedad mental, debido a que las técnicas de análisis empleadas no permiten separar los efectos específicos de cada una de las variables independientes.

Convendría añadir que el concepto de clase social lleva implicados muchos componentes de tipo psicológico, ya que las personas experimentan la clase social desde perspectivas muy distintas, de acuerdo con su propia posición dentro de la estructura de clases, y estas experiencias distintas inciden directamente sobre las respuestas que los entrevistados dan a las preguntas sobre clase social. En este sentido, si tenemos en cuenta la situación actual del problema, quizá sería mejor, tal como sugiere Morris (1964), que los psicólogos sociales y los sociólogos se dedicaran primero a hacer psicología social y sociología, estudiando a fondo conceptos tales como estratificación, movilidad social, cambio social, etc., y a continuación, una vez clarificados estos conceptos básicos, podrían ya aportar claridad al problema de los factores sociales y su incidencia sobre la enfermedad mental.

Salvo raras excepciones, los estudios epidemiológicos sobre la enfermedad mental hasta ahora han caído presa de muchas correlaciones bivariadas descubiertas en el curso de la investigación. Sin embargo, según dijimos más arriba, las técnicas multivariadas nos permiten aislar los efectos de cada variable independiente sobre la dependiente (enfermedad mental), así como la interrelación entre las variables independientes o predictoras y su impacto conjunto (interacción) sobre la variable dependiente.

Los problemas de la investigación sobre epidemiología psiquiátrica han sido muchos y han sido puestos de manifiesto una y otra vez en la bibliografía sobre la enfermedad mental. Mencionemos, por ejemplo, el fallo que supone el no distinguir claramente entre incidencia y prevalencia de la enfermedad mental en la investigación empírica (Kleiner y Parker, 1970; Dunham, 1964). Asimismo, tal como han destacado algunos autores, los índices utilizados en los estudios de campo son, cuando menos, sospechosos (Kohn, 1970; Dohrenwend y Dohrenwend, 1969; Plunkett y Gordon, 1960). Resultan también frecuentes los problemas con respecto a la medición de las 
variables (Elinson, 1972; Mechanic, 1969; Mischler y Scotch, 1970). No obstante, uno de los puntos claves de la epidemiología psiquiátrica es la cuestión, aún no resuelta, de definir qué es un caso psiquiátrico (Morris, 1964; Hollingshead, 1958; Milbank Memorial Fund, 1950; Blum, 1962; Kessel, 1962; Dunham, 1964; Mischler y Siotch, 1970).

Resulta difícil definir qué es un caso psiquiátrico a menos que se logre un acuerdo en definir qué es la enfermedad mental. En este sentido, las definiciones son o muy amplias o excesivamente limitadas (Dunham, 1964; Dohrenwend y Dohrenwend, 1969). Este aspecto hace que los distintos estudios realizados no sean comparables (Dohrenwend y Dohrenwend, 1969; Morris, 1964; Dunham, 1964; Rosenthal, 1970; Mischler y Scotch, 1970). Esta es la razón por la que Weakland ha acusado a muchos de estos estudios clásicos de atomísticos y anti-teoréticos.

Otra serie de problemas se deriva de la existencia de muchas fuentes de variabilidad de los procedimientos diagnósticos. En primer lugar, los psiquiatras varían en cuanto a sus preferencias diagnósticas: unos mismos síntomas pueden ser diagnosticados de forma distinta y con diferentes categorías psiquiátricas por distintos psiquiatras. Los pacientes, a su vez, varían en cuanto a calidad y cantidad de síntomas, en el sentido de que difieren en cuanto al número y calidad de los mismos, incluso dentro de la misma categoría diagnóstica. Finalmente, la interacción entre observador y sujeto admite también una variación considerable. Por esta razón, Weakland (1969) habla de auténticos «pufos» diagnósticos y de variabilidad de los índices empleados. Por su parte, Plunkett y Gordon (1960) destacan la existencia de modas en el diagnóstico.

Según ya hemos indicado, muchos de los conceptos utilizados en la investigación empírica resultan vagos y no nos dicen nada sobre la etiología (Dunham, 1970). En este sentido, Martin (1962) destaca el hecho de que el concepto de clase social resulta escurridizo, y de que los índices de educación poseen escaso valor debido a su distribución en la población. Se ha criticado asimismo el concepto de salud y enfermedad empleado por los distintos estudios (Elinson, 1972). Por otra parte, el concepto de esquizofrenia no resulta claramente reconocible y comprensible o, lo que es lo mismo, la categoría «esquizofrénico» no está claramente delimitada y definida. Cabe señalar también que, a veces, las variables sociales utilizadas son escasamente relevantes (Weakland, 1969). Ya hemos mencionado el hecho de que incluso el concepto mismo de etiología es frecuentemente malentendido (McKinlay, 1973).

Otra dificultad importante en los estudios que usan muestras y cuestionarios se refieren a los sesgos introducidos por la distancia social y los efectos del entrevistador, la influencia del sexo, así como las dificultades implícitas en todo el proceso de recolección de datos. El libro de McKinlay (1973) ofrece ejemplos claros y concretos de estos problemas básicos de 
la metodología de la investigación. Ahora bien, aun suponiendo que se hubieran controlado las dificultades que acabamos de mencionar, persiste todavía el grave problema de la fiabilidad y validez de los estudios de campo de la enfermedad mental (Dohrenwend y Dohrenwend, 1969 y 1970; Elinson, 1972; Dunham, 1961; Dunham, 1964). Esta falta de fiabilidad y validez podría explicar el hecho de que la dirección de la causalidad esté sujeta a debate y de que sean posibles distintas interpretaciones de los mismos estudios (Kohn, 1970; Dunham, 1964; Dohrenwend y Dohrenwend, 1969). Por la misma razón, sucede también que los investigadores obtienen, con frecuencia, resultados ambiguos o incluso contradictorios. Este panorama se complica todavía más por la presencia de correlaciones espúreas entre las variables utilizadas (Mischler y Scotch, 1970; Brenner, 1973), ya que a veces los autores deducen de una correlación alta y positiva entre determinadas variables una dependencia entre ellas, cuando, de hecho, la asociación entre dichas variables puede estar dependiendo de otra u otras variables no identificadas. Destaquemos finalmente el hecho de que si bien las críticas precedentes pueden resultar excesivas, sin embargo, sigue siendo cierto que la mayoría de los estudios de epidemiología psiquiátrica están sujetos a varias limitaciones metodológicas importantes que, en parte al menos, explican la falta de acuerdo con respecto a la relación entre variables sociales y disfunciones psicológicas.

\section{Perspectivas futuras}

De la discusión precedente se deduce la necesidad de una nueva metodología en los estudios futuros sobre la enfermedad mental. Necesitamos, asimismo, una mayor clarificación de los siguientes problemas: un análisis a fondo de la dinámica de la clase social y sus implicaciones psicológicas y de la dinámica de las disfunciones psicológicas y sus inter-relaciones con los procesos sociales (Fried, 1976). Urge, por otra parte, llevar a cabo enfoques interdisciplinares desde el punto de vista psicológico, social y psiquiátrico (Fried, 1970). A su vez, es conveniente una mayor clarificación de los factores psicosociales en cuanto variables intervinientes (Kleiner y Parker, 1970). Por otra parte, dado que la estructura de clases se ha convertido en un fenómeno muy complejo, no puede ser medida mediante un solo índice o escala; es necesario, pues, un enfoque pluralista (Martin, 1962:53). Habría que enfatizar, asimismo, la concepción del ajuste individuo-medio ambiente (Prince, 1974), analizándolo desde la perspectiva de la estructura del bienestar psicológico (Bradburn, 1969 y 1970). De hecho, habría que considerar la esquizofrenia como una conducta social más bien que patológica (Weakland, 1969:693), para evitar al máximo los procesos de etiquetación que, a su vez, suelen llevar a la estigmatización social de las personas. 
Convendría, finalmente, no olvidar que la etiología es más importante que la incidencia y la prevalencia ${ }^{2}$, ya que ambas están determinadas por el problema etiológico (Opler, 1967:45).

Podríamos concluir, en síntesis, que el cometido de la investigación futura sobre las relaciones entre estructura social y enfermedad mental habría de centrarse en las relaciones interpersonales, en los enfoques pluralistas y, sobre todo, en los aspectos de metodología de la investigación. En realidad, nuestra preferencia personal reside en los problemas metodológicos, ya que juzgamos necesario un mayor refinamiento de las variables a utilizar. El primer objetivo habría de consistir en obtener datos fiables y válidos. Por otra parte, un enfoque pluralista o de factores múltiples nos permitiría un estudio más realista de las disfunciones psicológicas, dado que la causa única o factor específico de la etiología no tiene en cuenta la complejidad de las disfunciones o trastornos psicológicos. En resumen, habría que desarrollar una Sociología de la Salud Mental ${ }^{3}$ que hiciera uso de los descubrimientos tradicionales con un punto de vista más crítico y pluridisciplinar.

2 Sobre el significado de los índices de incidencia y prevalencia, véase J. M. DE Miguel (1973) y (1978).

${ }^{3}$ Una introducción teórica al tema de la sociología de la salud mental puede verse en J. M. DE MIGUEL (1978), cap. 3, y en (1976). Con respecto a la infraestructura del sistema psiquiátrico español, véase B. González (1978), OLtra, De Mrguel (1971) y MONTOYa (1978). 